\title{
OPERATOR SPLITTING FOR PARTIAL DIFFERENTIAL EQUATIONS WITH BURGERS NONLINEARITY
}

\author{
HELGE HOLDEN, CHRISTIAN LUBICH, AND NILS HENRIK RISEBRO
}

\begin{abstract}
We provide a new analytical approach to operator splitting for equations of the type $u_{t}=A u+u u_{x}$ where $A$ is a linear differential operator such that the equation is well-posed. Particular examples include the viscous Burgers equation, the Korteweg-de Vries (KdV) equation, the Benney-Lin equation, and the Kawahara equation. We show that the Strang splitting method converges with the expected rate if the initial data are sufficiently regular. In particular, for the $\mathrm{KdV}$ equation we obtain second-order convergence in $H^{r}$ for initial data in $H^{r+5}$ with arbitrary $r \geq 1$.
\end{abstract}

\section{INTRODUCTION}

In this paper we study initial value problems of the type

$$
u_{t}=P\left(\partial_{x}\right) u+u u_{x} \quad(x \in \mathbb{R}, 0 \leq t \leq T),\left.\quad u\right|_{t=t_{0}}=u_{0},
$$

with a polynomial $P$ of degree $\ell \geq 2$ satisfying

$$
\operatorname{Re} P(i \xi) \leq 0 \quad \text { for all } \xi \in \mathbb{R} .
$$

This class includes several important equations like

$$
\begin{array}{ll}
u_{t}=u_{x x}+u u_{x}, & \text { the viscous Burgers equation, } \\
u_{t}=u_{x x x}+u u_{x}, & \text { the Korteweg-de Vries equation, } \\
u_{t}=-u_{x x x}-\beta\left(u_{x x}+u_{x x x x}\right)-u_{x x x x x}+u u_{x}, & \text { the Benney-Lin equation [2, 15], } \\
u_{t}=u_{x x x x x}-u_{x x x}+u u_{x}, & \text { the Kawahara equation [12]. }
\end{array}
$$

We employ operator splitting (see, e.g., 6]), i.e., construction of an approximate solution by concatenating the solutions of the separate problems

$$
v_{t}=P\left(\partial_{x}\right) v \text { and } \quad w_{t}=w w_{x} .
$$

More precisely, if we let $u(t)=\Phi_{C}^{t}\left(u_{0}\right)$ denote the solution of the initial value problem

$$
u_{t}=C(u),\left.\quad u\right|_{t=0}=u_{0},
$$

then (sequential) operator splitting claims that the exact solution $u(t)=\Phi_{C}^{t}\left(u_{0}\right)$ is well approximated by $u_{n}$, at $t=n \Delta t \leq T$ and as $\Delta t \rightarrow 0$, where

$$
u_{n+1}=\Phi_{A}^{\Delta t}\left(\Phi_{B}^{\Delta t}\left(u_{n}\right)\right), \quad n=0,1,2, \ldots,
$$

Received by the editor February 8, 2011.

2010 Mathematics Subject Classification. Primary 35Q53; Secondary: 65M12, 65M15.

Key words and phrases. Operator splitting, Burgers equation, KdV equation, Benney-Lin equation, Kawahara equation.

This work was supported in part by the Research Council of Norway.

(C)2012 American Mathematical Society Reverts to public domain 28 years from publication 
and $C=A+B$. In our case we have the operator

$$
A u=P\left(\partial_{x}\right) u
$$

and $B$ will be the Burgers operator

$$
B(u)=u u_{x}
$$

acting on appropriate Sobolev spaces. In the present paper a more refined operator splitting, known as Strang splitting, will be discussed. Strang splitting means that we consider the approximation

$$
u_{n+1}=\Psi^{\Delta t}\left(u_{n}\right)=\Phi_{A}^{\Delta t / 2} \circ \Phi_{B}^{\Delta t} \circ \Phi_{A}^{\Delta t / 2}\left(u_{n}\right), \quad n=0,1,2, \ldots .
$$

The technique we use requires a well-posedness theory for the full equation in Sobolev spaces. This is currently available for the equations listed above, see [14] (the viscous Burgers equation), 4, 13, 16] (the KdV equation), 3] (the BenneyLin equation), and [11] (the Kawahara equation). The challenge in using operator splitting for the present class of equations is the fact that the Burgers step, i.e., solving the second equation in (1.3), generically introduces singularities in finite time irrespective of the smoothness of the initial data, while the solution of the full problem (1.4) remains smooth. Thus the determination of the time step used in the operator splitting and the control of the smoothness of the approximate solution are rather delicate. This implies that the standard estimates that are required for operator splitting (see, e.g., [6]) do not apply. The idea of applying operator splitting to the KdV equation was introduced in a short note by Tappert [19], with numerical tests. A further study was done in [7] where a Lax-Wendroff theorem was proved, namely assuming convergence of operator splitting, one can prove that the limit is a weak solution of the KdV equation. Furthermore, extensive numerical computations were presented, all indicating that operator splitting does converge. A completely different and novel approach was taken in the paper [8]. Previous results had as a goal to use operator splitting to show existence of solutions of the $\mathrm{KdV}$ equation. However, in [8] one took for granted the well-posedness of the Cauchy problem for the KdV equation in Sobolev spaces of high order. By using a bootstrap principle (see [18]), one could show that the Burgers step in a Sobolev space of lower order would avoid blow-up, thereby securing that the full approximation remained smooth, and indeed converged to the solution of the KdV equation. A key part of this analysis was to secure a uniform time step in the approximation. The approach in [8] also presented a new interpolation between the discrete time steps based on the introduction of a new auxiliary time variable, and hence a two-variable approximation was used.

In the present paper we take a different approach. We do take as a starting point the well-posedness of the Cauchy problem for the KdV equation, and we use the same analysis to estimate carefully the change in Sobolev norm during the Burgers step. However, instead of introducing a two-variable approximation and applying the bootstrap principle, we perform an analysis that identifies the principal error terms of the local error as quadrature errors, which are estimated via bounds of Lie commutators. Such a type of analysis was first done for operator splitting for linear evolution equations in [10] and for nonlinear Schrödinger equations in [17]. The error propagation is studied via "Lady Windermere's fan" [5], as is usual in the error analysis of time discretization methods, controlling here carefully 
the spatial regularity of the numerical approximations by first establishing lowerorder convergence in a higher-order Sobolev norm, as in [17. In this way we improve and simplify the results in [8]. The main result says that the Strang splitting converges to the full solution with the expected second-order convergence rate, in the $H^{r}$ Sobolev norm for $r \geq 1$ in the case of $H^{r+5}$ initial data for the $\mathrm{KdV}$ equation. Furthermore, as was clear already in [8, the analysis extends to a class of dispersive equations, and here we find it suitable to study the class (1.1) subject to the condition (1.2). This means that the equations all share a Burgers nonlinearity. The extension of the present approach to dispersive equations with other nonlinearities, not necessarily quadratic and also in higher spatial dimensions, appears feasible as long as an analogue of the regularity properties of the Burgers step can be established. We also refer to [9] where the approach of $[8]$ is applied to the generalized active scalar equation

$$
\theta_{t}+\boldsymbol{u} \cdot \nabla \theta+\Lambda^{\alpha} \theta=0, \quad \boldsymbol{u}=\operatorname{curl} \Lambda^{-\beta} \theta,
$$

where $\nabla$ is the spatial gradient operator and $\Lambda=(-\Delta)^{1 / 2}$. The unknown function is $\theta:[0, T] \times \mathbb{R}^{2} \rightarrow \mathbb{R}$ and we assume $\alpha \in(0,2]$.

\section{ERror bounds for Strang SPlitting: StATEMENT of RESUlts}

Let $r$ be a positive integer and associate with it

$$
p=r+2 \ell-1, \quad q=r+\ell-1=p-\ell,
$$

where $\ell \geq 2$ is the degree of the polynomial $P$ in (1.1). The integers $p, q$ and $r$ will be kept fixed throughout the paper.

We require local well-posedness of (1.1) in $H^{r}$ and $H^{q}$ in the following sense: For both $s=r$ and $s=q$ and for a given time $T$, there exists $R>0$ such for all $u_{0} \in H^{s}$ with $\left\|u_{0}\right\|_{H^{s}} \leq R$, there is a unique strong solution $u \in C\left([0, T], H^{s}\right)$ of (1.1) with initial data $u_{0}$, and the dependence on the initial data is locally Lipschitzcontinuous: There is a constant $K=K(R, T)<\infty$ such that the solutions $\widetilde{u}, u$ corresponding to arbitrary initial data $\widetilde{u}_{0}, u_{0}$ in the $H^{s}$-ball of radius $R$ satisfy

$$
\|\widetilde{u}(t)-u(t)\|_{H^{s}} \leq K\left\|\widetilde{u}_{0}-u_{0}\right\|_{H^{s}} \quad \text { for } \quad 0 \leq t \leq T \text {. }
$$

For the KdV equation, it is known from [13] that this well-posedness assumption holds for every $s \in \mathbb{N}$, for arbitrary final time $T$ and with arbitrary positive $R$.

For the general class (1.1) and (1.2), it can be shown with arguments as in Lemma 3.2 below, that the above well-posedness result holds, at least, with sufficiently small $R=R(T)$ for any given $T$, or for sufficiently small $T=T(R)$ for any given $R$, the same as for the inviscid Burgers equation. For the equations listed in the introduction, however, the well-posedness situation is more favorable than for the inviscid Burgers equation; see [14] (the viscous Burgers equation), 4, 13, 16] (the KdV equation), 3] (the Benney-Lin equation), and [11] (the Kawahara equation).

We consider solutions bounded by

$$
\|u(t)\|_{H^{p}} \leq \rho<R \quad \text { for } 0 \leq t \leq T .
$$

In particular, we assume that the initial data are in $H^{p}$ :

$$
u_{0} \in H^{p} .
$$

Under these assumptions we will in this paper show the following results for the Strang splitting (1.8). 
Theorem 2.1 (First-order convergence in $H^{q}$ and bound in $H^{p}$ ). There is $\overline{\Delta t}>0$ such that for $\Delta t \leq \overline{\Delta t}$ and $t_{n}=n \Delta t \leq T$,

$$
\left\|u_{n}-u\left(t_{n}\right)\right\|_{H^{q}} \leq C_{1} \Delta t \quad \text { and } \quad\left\|u_{n}\right\|_{H^{p}} \leq C_{0} .
$$

Here, $\overline{\Delta t}, C_{0}$ and $C_{1}$ only depend on $\left\|u_{0}\right\|_{H^{p}}, \rho$, and $T$.

This result will be used to prove our main result:

Theorem 2.2 (Second-order convergence in $H^{r}$ ). Assume that we have a solution $u$ of (1.1) that satisfies (2.2). Define the Strang approximation $u_{n}$ by (1.8). Then the following statement holds: There is $\overline{\Delta t}>0$ such that for $\Delta t \leq \overline{\Delta t}$ and $t_{n}=$ $n \Delta t \leq T$,

$$
\left\|u_{n}-u\left(t_{n}\right)\right\|_{H^{r}} \leq C_{2} \Delta t^{2} .
$$

Here, $\overline{\Delta t}$ and $C_{2}$ only depend on $\left\|u_{0}\right\|_{H^{p}}, \rho$, and $T$.

For the $\mathrm{KdV}$ equation, this result yields second-order convergence in $H^{r}$ for initial data in $H^{r+5}$ for arbitrary $r \in \mathbb{N}$. This improves significantly on the result of [8], where second-order convergence is shown in the $H^{r}$-norm for $r \geq 8$ for initial data in $H^{r+9}$. The proof uses a regularity result for the inviscid Burgers equation as in [8], but is otherwise quite different from the proof given there. It is conceptually closely related to the error analysis of Strang splitting given in [17 for the nonlinear Schrödinger equation. The proofs of Theorems 2.1 and 2.2 will cover the remainder of the paper. We remark that throughout our computations, $C$ denotes a generic constant whose value may change at each occurrence.

\section{Regularity Results For the inViscid Burgers Equation}

The following variant of a result in [8] will play a key role in the proof of Theorem 2.1

Lemma 3.1. If $\left\|\Phi_{B}^{t}\left(u_{0}\right)\right\|_{H^{q}} \leq \alpha$ for $0 \leq t \leq \Delta t$, then $\left\|\Phi_{B}^{t}\left(u_{0}\right)\right\|_{H^{p}} \leq e^{c \alpha t}\left\|u_{0}\right\|_{H^{p}}$ for $0 \leq t \leq \Delta t$, where $c$ is independent of $u_{0}$ and $\Delta t$.

Proof. We find that $w(t)=\Phi_{B}^{t}\left(u_{0}\right)$ satisfies (cf. [8])

$$
\begin{aligned}
& \|w\|_{H^{p}} \frac{d}{d t}\|w\|_{H^{p}}=\frac{1}{2} \frac{d}{d t}\left\|\Phi_{B}^{t}\left(u_{0}\right)\right\|_{H^{p}}^{2}=\left(w, w_{t}\right)_{H^{p}} \\
& =\sum_{j=0}^{p} \int_{\mathbb{R}} \partial_{x}^{j} w \partial_{x}^{j}\left(w w_{x}\right) d x=\sum_{j=0}^{p} \sum_{k=0}^{j}\left(\begin{array}{l}
j \\
k
\end{array}\right) \int \partial_{x}^{j} w \partial_{x}^{k+1} w \partial_{x}^{j-k} w d x .
\end{aligned}
$$

For $j<p$, any of the above terms can be estimated by

$$
\begin{aligned}
\left|\int \partial_{x}^{j} w \partial_{x}^{k+1} w \partial_{x}^{j-k} w d x\right| & \leq\left\|\partial_{x}^{j} w\right\|_{L^{\infty}}\left\|\partial_{x}^{\max \{k+1, j-k\}} w\right\|_{L^{2}}\left\|\partial_{x}^{\min \{k+1, j-k\}} w\right\|_{L^{2}} \\
& \leq C\|w\|_{H^{p}}^{2}\|w\|_{H^{q}} \\
& \leq C \alpha\|w\|_{H^{p}}^{2},
\end{aligned}
$$

by using the Sobolev inequality $\|v\|_{L^{\infty}} \leq \frac{1}{\sqrt{2}}\|v\|_{H^{1}}$ (so that $C=1 / \sqrt{2}$ ) and the fact that $\min \{k+1, j-k\} \leq \frac{1}{2}(j+1) \leq \frac{1}{2} p \leq p-\ell=q$ since $p \geq 2 \ell$. For $j=p$ we estimate for $k \leq q$ and $k \neq q-1$ by

$$
I:=\left|\int \partial_{x}^{p} w \partial_{x}^{k+1} w \partial_{x}^{p-k} w d x\right| \leq\left\|\partial_{x}^{p} w\right\|_{L^{2}}\left\|\partial_{x}^{k+1} w\right\|_{L^{\infty}}\left\|\partial_{x}^{p-k} w\right\|_{L^{2}}
$$




$$
\leq C\|w\|_{H^{p}}\|w\|_{H^{k+2}}\|w\|_{H^{p-k}} .
$$

To estimate this further we have to distinguish the cases $k+2 \leq q$ and $k=q$. In the former case we find

$$
I \leq C\|w\|_{H^{p}}\|w\|_{H^{q}}\|w\|_{H^{p}} \leq C \alpha\|w\|_{H^{p}}^{2},
$$

while in the latter case we obtain

$$
I \leq C\|w\|_{H^{p}}\|w\|_{H^{p}}\|w\|_{H^{q}} \leq C \alpha\|w\|_{H^{p}}^{2},
$$

since $q+2 \leq q+\ell=p$ and $p-q=\ell \leq \ell+r-1=q$.

In the two cases where either $k+1=q$ or $q+2 \leq k+1 \leq p$ we estimate

$$
\begin{aligned}
I & \leq\left\|\partial_{x}^{p} w\right\|_{L^{2}}\left\|\partial_{x}^{k+1} w\right\|_{L^{2}}\left\|\partial_{x}^{p-k} w\right\|_{L^{\infty}} \\
& \leq C\|w\|_{H^{p}}\|w\|_{H^{k+1}}\|w\|_{H^{p-k+1}} .
\end{aligned}
$$

In both cases this is bounded by $C \alpha\|w\|_{H^{p}}^{2}$, since in the case $k+1=q$ we have $p-k+1=\ell+2 \leq 2 \ell \leq p$, and for $k+1 \geq q+2$ we have $p-k+1 \leq p-q=\ell \leq q$.

We are left with the term where $k=p=j$, viz.

$$
\begin{aligned}
\left|\int \partial_{x}^{p} w \partial_{x}^{p+1} w w d x\right| & =\frac{1}{2}\left|\int\left(\partial_{x}^{p} w\right)^{2} \partial_{x} w d x\right| \\
& \leq\left\|\partial_{x} w\right\|_{L^{\infty}}\left\|\partial_{x}^{p} w\right\|_{L^{2}}^{2} \\
& \leq C\|w\|_{H^{q}}\|w\|_{H^{p}}^{2}
\end{aligned}
$$

since $q \geq \ell \geq 2$. Thus,

which concludes the proof.

$$
\frac{d}{d t}\|w(t)\|_{H^{p}} \leq c \alpha\|w(t)\|_{H^{p}}
$$

We also need the following lemma:

Lemma 3.2. If $\left\|u_{0}\right\|_{H^{m}} \leq M$ for some $m \geq 1$, then there exists $\bar{t}(M)>0$ such that $\left\|\Phi_{B}^{t}\left(u_{0}\right)\right\|_{H^{m}} \leq 2 M$ for $0 \leq t \leq \bar{t}(M)$.

Proof. The calculation (3.1) with $m$ instead of $p$, and the subsequent arguments, show that

$$
\|w(t)\|_{H^{m}} \frac{d}{d t}\|w(t)\|_{H^{m}} \leq c\|w(t)\|_{H^{m}}^{3},
$$

and hence the result follows by comparing with the majorizing differential equation $y^{\prime}=c y^{2}$.

Finally, we will make use of the following regularity result.

Lemma 3.3. If $\left\|u_{0}\right\|_{H^{p}} \leq C_{0}$, then there exists $\bar{t}$ depending only on $C_{0}$, such that the solution of the inviscid Burgers equation with initial data $u_{0}, w(t)=\Phi_{B}^{t}\left(u_{0}\right)$, satisfies

$$
w \in C^{2}\left([0, \bar{t}], H^{q}\right) \quad \text { and } \quad w \in C^{3}\left([0, \bar{t}], H^{r}\right) .
$$

Proof. If we define, for $t \in[0, \bar{t}]$ with $\bar{t}$ from Lemma 3.2 for $m=p$,

$$
\widetilde{w}(t)=u_{0}+t B\left(u_{0}\right)+\int_{0}^{t}(t-s) d B(w(s))[B(w(s))] d s
$$

and not $11 d B(w)[B(w)]=w^{2} w_{x x}+2 w w_{x}^{2}$, then we have $\widetilde{w} \in C^{2}\left([0, \bar{t}], H^{q}\right)$. Since $\widetilde{w}_{t t}=d B(w)[B(w)]=B(w)_{t}$ and $\widetilde{w}_{t}(0)=B\left(u_{0}\right)=w_{t}(0)$ and $\widetilde{w}(0)=u_{0}=w(0)$,

\footnotetext{
${ }^{1}$ We adopt the notation from 1 .
} 
we have $\widetilde{w}=w$. The second statement is proved in the same way, by computing $\widetilde{w}_{t t t}$.

\section{LOCAL ERROR IN $H^{q}$}

Lemma 4.1. The local error of the Strang splitting is bounded in $H^{q}$ by

$$
\left\|\Psi^{\Delta t}\left(u_{0}\right)-\Phi_{A+B}^{\Delta t}\left(u_{0}\right)\right\|_{H^{q}} \leq c_{1} \Delta t^{2},
$$

where $c_{1}$ only depends on $\left\|u_{0}\right\|_{H^{p}}$.

Proof. The proof follows that of the local error bound in [17, §4.4]. We write $e^{t A} v=\Phi_{A}^{t}(v)$ to indicate the linearity of the flow of $A$. We start from the variationof-constants formula for $u(t)=\Phi_{A+B}^{t}\left(u_{0}\right)$,

$$
u(t)=e^{t A} u_{0}+\int_{0}^{t} e^{(t-s) A} B(u(s)) d s,
$$

which is just the formula $\phi(t)-\phi(0)=\int_{0}^{t} \dot{\phi}(s) d s$ for $\phi(s)=e^{(t-s) A} u(s)$, and its sibling formula

$$
B(u(s))=B\left(e^{s A} u_{0}\right)+\int_{0}^{s} d B\left(e^{(s-\sigma) A} u(\sigma)\right)\left[e^{(s-\sigma) A} B(u(\sigma))\right] d \sigma,
$$

which is nothing but the formula $B(\varphi(s))-B(\varphi(0))=\int_{0}^{s} d B(\varphi(\sigma))[\dot{\varphi}(\sigma)] d \sigma$ for $\varphi(\sigma)=e^{(s-\sigma) A} u(\sigma)$. We insert (4.2) into (4.1) with $t=\Delta t$ to obtain

$$
u(\Delta t)=e^{\Delta t A} u_{0}+\int_{0}^{\Delta t} e^{(\Delta t-s) A} B\left(e^{s A} u_{0}\right) d s+e_{1}
$$

with

$$
e_{1}=\int_{0}^{\Delta t} \int_{0}^{s} e^{(\Delta t-s) A} d B\left(e^{(s-\sigma) A} u(\sigma)\right)\left[e^{(s-\sigma) A} B(u(\sigma))\right] d \sigma d s .
$$

On the other hand, for the result after one step of the Strang splitting,

$$
u_{1}=\Psi^{\Delta t}\left(u_{0}\right)=e^{\Delta t A / 2} \Phi_{B}^{\Delta t}\left(e^{\Delta t A / 2} u_{0}\right),
$$

we use the first-order Taylor expansion with integral remainder term in $H^{q}$,

$$
\Phi_{B}^{\Delta t}(v)=v+\Delta t B(v)+\Delta t^{2} \int_{0}^{1}(1-\theta) d B\left(\Phi_{B}^{\theta \Delta t}(v)\right)\left[B\left(\Phi_{B}^{\theta \Delta t}(v)\right)\right] d \theta .
$$

This is justified for $v=e^{\Delta t A / 2} u_{0} \in H^{p}$ and for sufficiently small $\Delta t$ by Lemma 3.3 . We therefore obtain

$$
u_{1}=e^{\Delta t A} u_{0}+\Delta t e^{\Delta t A / 2} B\left(e^{\Delta t A / 2} u_{0}\right)+e_{2}
$$

with

$$
e_{2}=\Delta t^{2} \int_{0}^{1}(1-\theta) e^{\Delta t A / 2} d B\left(\Phi_{B}^{\theta \Delta t}\left(e^{\Delta t A / 2} u_{0}\right)\right)\left[B\left(\Phi_{B}^{\theta \Delta t}\left(e^{\Delta t A / 2} u_{0}\right)\right)\right] d \theta .
$$

The error thus becomes

$$
u_{1}-u(\Delta t)=\Delta t e^{\Delta t A / 2} B\left(e^{\Delta t A / 2} u_{0}\right)-\int_{0}^{\Delta t} e^{(\Delta t-s) A} B\left(e^{s A} u_{0}\right) d s+\left(e_{2}-e_{1}\right),
$$

and hence the principal error term is just the quadrature error of the midpoint rule applied to the integral over $[0, \Delta t]$ of the function

$$
f(s)=e^{(\Delta t-s) A} B\left(e^{s A} u_{0}\right) .
$$


We express the quadrature error in first-order Peano form,

$$
\Delta t f\left(\frac{1}{2} \Delta t\right)-\int_{0}^{\Delta t} f(s) d s=\Delta t^{2} \int_{0}^{1} \kappa_{1}(\theta) f^{\prime}(\theta \Delta t) d \theta
$$

where $\kappa_{1}$ is the real-valued, bounded Peano kernel of the midpoint rule. We find

$$
f^{\prime}(s)=-e^{(\Delta t-s) A}[A, B]\left(e^{s A} u_{0}\right)
$$

with the Lie commutator

$$
[A, B](v)=d A(v)[B(v)]-d B(v)[A v]=P\left(\partial_{x}\right)\left(v v_{x}\right)-\left(P\left(\partial_{x}\right) v\right) v_{x}-v P\left(\partial_{x}\right) v_{x} .
$$

We have that $P$ is linear in $\partial_{x}^{k}$ for $k \leq \ell$, and we compute

$$
\begin{aligned}
\left(\partial_{x}^{\ell}\right)\left(v v_{x}\right)-\left(\partial_{x}^{\ell} v\right) v_{x}-v \partial_{x}^{\ell} v_{x} & =\sum_{k=0}^{\ell}\left(\begin{array}{l}
\ell \\
k
\end{array}\right) \partial_{x}^{k} v \partial_{x}^{\ell+1-k} v-\left(\partial_{x}^{\ell} v\right) v_{x}-v \partial_{x}^{\ell+1} v \\
& =\sum_{k=1}^{\ell-1}\left(\begin{array}{l}
\ell \\
k
\end{array}\right) \partial_{x}^{k} v \partial_{x}^{\ell+1-k} v .
\end{aligned}
$$

Hence the terms containing derivatives of order $\ell+1$ disappear, and we obtain the commutator bound

$$
\|[A, B](v)\|_{H^{q}} \leq C\left(\|v\|_{L^{\infty}}\|v\|_{H^{p}}+\left\|v_{x}\right\|_{L^{\infty}}\|v\|_{H^{p}}+\|v\|_{H^{p-1}}^{2}\right) \leq C\|v\|_{H^{p}}^{2} .
$$

Since $e^{t A}$ does not increase the Sobolev norms, it follows that

$$
\left\|f^{\prime}(s)\right\|_{H^{q}} \leq C\left\|u_{0}\right\|_{H^{p}}^{2}
$$

and hence the quadrature error is $O\left(\Delta t^{2}\right)$ in the $H^{q}$ norm for $u_{0} \in H^{p}$. The $H^{q}$ norm of the remainder term $e_{2}-e_{1}$ is bounded by $C \Delta t^{2}$ for $u_{0} \in H^{p}$ for sufficiently small $\Delta t$ (by using Lemma 3.2). We include the details for the convenience of the reader:

$$
\begin{aligned}
\left\|e_{1}\right\|_{H^{q}} & \leq \int_{0}^{\Delta t} \int_{0}^{s}\left\|e^{(\Delta t-s) A} d B\left(e^{(s-\sigma) A} u(\sigma)\right)\left[e^{(s-\sigma) A} B(u(\sigma))\right]\right\|_{H^{q}} d \sigma d s \\
& \leq \int_{0}^{\Delta t} \int_{0}^{s}\left\|\left(\left(e^{(s-\sigma) A} u(\sigma)\right)\left(e^{(s-\sigma) A} B(u(\sigma))\right)\right)_{x}\right\|_{H^{q}} d \sigma d s \\
& \leq C \int_{0}^{\Delta t} \int_{0}^{s}\|u(\sigma)\|_{H^{q+1}}\|B(u(\sigma))\|_{H^{q+1}} d \sigma d s \\
& \leq C \int_{0}^{\Delta t} \int_{0}^{s}\|u(\sigma)\|_{H^{q+1}}\|u(\sigma)\|_{H^{q+1}}\|u(\sigma)\|_{H^{q+2}} d \sigma d s \\
& \leq C \Delta t^{2} R^{3}
\end{aligned}
$$

and

$$
\begin{aligned}
\left\|e_{2}\right\|_{H^{q}} & \leq \Delta t^{2} \int_{0}^{1}(1-\theta)\left\|e^{\Delta t A / 2} d B\left(\Phi_{B}^{\theta \Delta t}\left(e^{\Delta t A / 2} u_{0}\right)\right)\left[B\left(\Phi_{B}^{\theta \Delta t}\left(e^{\Delta t A / 2} u_{0}\right)\right)\right]\right\|_{H^{q}} d \theta \\
& \leq \Delta t^{2} \int_{0}^{1}\left\|\left(\left(\Phi_{B}^{\theta \Delta t}\left(e^{\Delta t A / 2} u_{0}\right)\right)\left(B\left(\Phi_{B}^{\theta \Delta t}\left(e^{\Delta t A / 2} u_{0}\right)\right)\right)\right)_{x}\right\|_{H^{q}} d \theta \\
& \leq \Delta t^{2} C \int_{0}^{1}\left\|\Phi_{B}^{\theta \Delta t}\left(e^{\Delta t A / 2} u_{0}\right)\right\|_{H^{q+1}}\left\|B\left(\Phi_{B}^{\theta \Delta t}\left(e^{\Delta t A / 2} u_{0}\right)\right)\right\|_{H^{q+1}} d \theta
\end{aligned}
$$




$$
\begin{aligned}
& \leq \Delta t^{2} C \int_{0}^{1}\left\|\Phi_{B}^{\theta \Delta t}\left(e^{\Delta t A / 2} u_{0}\right)\right\|_{H^{q+1}}^{2}\left\|\Phi_{B}^{\theta \Delta t}\left(e^{\Delta t A / 2} u_{0}\right)\right\|_{H^{q+2}} d \theta \\
& \leq C \Delta t^{2} R^{3} .
\end{aligned}
$$

Thus the proof is complete.

\section{Proof of Theorem 2.1}

The proof uses "Lady Windermere's fan" with error propagation by the exact flow [5, p. 160, Fig. 3.1]. In our approach the necessary regularity for estimating local errors by Lemma 4.1 is ensured by Lemma 3.1 via an induction argument, which we present next.

We make the induction hypothesis that for $k \leq n-1$,

$$
\begin{aligned}
& \left\|u_{k}\right\|_{H^{q}} \leq R, \quad\left\|u_{k}\right\|_{H^{p}} \leq e^{2 c R k \Delta t}\left\|u_{0}\right\|_{H^{p}} \leq C_{0}, \\
& \left\|u_{k}-u\left(t_{k}\right)\right\|_{H^{q}} \leq \gamma \Delta t,
\end{aligned}
$$

where $C_{0}=e^{2 c R T}\left\|u_{0}\right\|_{H^{p}}$ with $c$ from Lemma 3.1 and $\gamma=K(R, T) c_{1}\left(C_{0}\right) T$ with $K(R, T)$ from the local Lipschitz bound (2.1) and $c_{1}\left(C_{0}\right)$ is the constant of Lemma 4.1 for starting values bounded by $C_{0}$ in $H^{p}$. We then show that the above bounds also hold for $k=n$ as long as $n \Delta t \leq T$ and $\Delta t$ is sufficiently small.

We denote, with $\Phi^{t}=\Phi_{A+B}^{t}$ for brevity,

$$
u_{n}^{k}=\Phi^{(n-k) \Delta t}\left(u_{k}\right),
$$

which is the value at time $t_{n}$ of the exact solution of (1.1) starting with initial data $u_{k}$ at time $t_{k}$. We note

We estimate

$$
u_{n}=u_{n}^{n}, \quad u\left(t_{n}\right)=u_{n}^{0}
$$

$$
\begin{aligned}
\left\|u_{n}-u\left(t_{n}\right)\right\|_{H^{q}} & \leq \sum_{k=0}^{n-1}\left\|u_{n}^{k+1}-u_{n}^{k}\right\|_{H^{q}} \\
& =\sum_{k=0}^{n-1}\left\|\Phi^{(n-k-1) \Delta t}\left(\Psi^{\Delta t}\left(u_{k}\right)\right)-\Phi^{(n-k-1) \Delta t}\left(\Phi^{\Delta t}\left(u_{k}\right)\right)\right\|_{H^{q}} .
\end{aligned}
$$

For $k \leq n-2$ we have $\left\|\Psi^{\Delta t}\left(u_{k}\right)\right\|_{H^{q}}=\left\|u_{k+1}\right\|_{H^{q}} \leq R$, and

$$
\begin{aligned}
\left\|\Phi^{\Delta t}\left(u_{k}\right)\right\|_{H^{q}} & \leq\left\|\Phi^{\Delta t}\left(u_{k}\right)-\Phi^{\Delta t}\left(u\left(t_{k}\right)\right)\right\|_{H^{q}}+\left\|\Phi^{\Delta t}\left(u\left(t_{k}\right)\right)\right\|_{H^{q}} \\
& \leq K(R, \Delta t)\left\|u_{k}-u\left(t_{k}\right)\right\|_{H^{q}}+\left\|u\left(t_{k+1}\right)\right\|_{H^{q}} \\
& \leq K(R, \Delta t) \gamma \Delta t+\rho,
\end{aligned}
$$

which is bounded by $R$ if $\Delta t$ is so small that

$$
K(R, \Delta t) \gamma \Delta t \leq R-\rho .
$$

Using (2.1) and Lemma 4.1 we therefore have, for $k \leq n-1$ and $n \Delta t \leq T$,

$$
\begin{aligned}
& \left\|\Phi^{(n-k-1) \Delta t}\left(\Psi^{\Delta t}\left(u_{k}\right)\right)-\Phi^{(n-k-1) \Delta t}\left(\Phi^{\Delta t}\left(u_{k}\right)\right)\right\|_{H^{q}} \\
& \quad \leq K(R, T)\left\|\Psi^{\Delta t}\left(u_{k}\right)-\Phi^{\Delta t}\left(u_{k}\right)\right\|_{H^{q}} \\
& \quad \leq K(R, T) c_{1}\left(C_{0}\right) \Delta t^{2} .
\end{aligned}
$$

With this estimate we obtain, again noting $n \Delta t \leq T$,

$$
\left\|u_{n}-u\left(t_{n}\right)\right\|_{H^{q}} \leq n K(R, T) c_{1}\left(C_{0}\right) \Delta t^{2} \leq \gamma \Delta t .
$$


With $\gamma \Delta t \leq R-\rho$, we have

$$
\left\|u_{n}\right\|_{H^{q}} \leq R
$$

Since $\left\|\Phi_{A}^{t}(v)\right\|_{H^{p}} \leq\|v\|_{H^{p}}$, Lemmas 3.1 and 3.2 yield, for $\Delta t \leq \bar{t}(R)$,

$$
\left\|u_{n}\right\|_{H^{p}}=\left\|\Phi_{A}^{\Delta t / 2} \circ \Phi_{B}^{\Delta t} \circ \Phi_{A}^{\Delta t / 2}\left(u_{n-1}\right)\right\|_{H^{p}} \leq e^{2 c R \Delta t}\left\|u_{n-1}\right\|_{H^{p}} \leq e^{2 c R n \Delta t}\left\|u_{0}\right\|_{H^{p}} .
$$

This completes the proof of Theorem 2.1

\section{LOCAL ERROR IN $H^{r}$}

Lemma 6.1. The local error of the Strang splitting is bounded in $H^{r}$ by

$$
\left\|\Psi^{\Delta t}\left(u_{0}\right)-\Phi_{A+B}^{\Delta t}\left(u_{0}\right)\right\|_{H^{r}} \leq c_{2} \Delta t^{3}
$$

where $c_{2}$ only depends on $\left\|u_{0}\right\|_{H^{p}}$.

Proof. The proof follows the lines of [17, §5.2]. Instead of (4.4) we now use the second-order Taylor expansion

$$
\begin{array}{rl}
\Phi_{B}^{\Delta t}(v)=v+\Delta t B(v)+\frac{1}{2} \Delta t^{2} & d B(v)[B(v)] \\
+\Delta t^{3} \int_{0}^{1} \frac{1}{2}(1-\theta)^{2}( & d^{2} B\left(\Phi_{B}^{\theta \Delta t}(v)\right)\left[B\left(\Phi_{B}^{\theta \Delta t}(v)\right), B\left(\Phi_{B}^{\theta \Delta t}(v)\right)\right] \\
& \left.+d B\left(\Phi_{B}^{\theta \Delta t}(v)\right)\left[d B\left(\Phi_{B}^{\theta \Delta t}(v)\right)\left[B\left(\Phi_{B}^{\theta \Delta t}(v)\right)\right]\right]\right) d \theta
\end{array}
$$

where henceforth we abbreviate the integral remainder term as

$$
\Delta t^{3} \int_{0}^{1} \frac{1}{2}(1-\theta)^{2}\left(d^{2} B(B, B)+d B d B B\right)\left(\Phi_{B}^{\theta \Delta t}(v)\right) d \theta .
$$

Hence,

$$
\begin{aligned}
u_{1}= & e^{\Delta t A} u_{0}+\Delta t e^{\Delta t A / 2} B\left(e^{\Delta t A / 2} u_{0}\right)+\frac{1}{2} \Delta t^{2} e^{\Delta t A / 2} d B\left(e^{\Delta t A / 2} u_{0}\right)\left[B\left(e^{\Delta t A / 2} u_{0}\right)\right] \\
& +\Delta t^{3} \int_{0}^{1} \frac{1}{2}(1-\theta)^{2} e^{\Delta t A / 2}\left(d^{2} B(B, B)+d B d B B\right)\left(\Phi_{B}^{\theta \Delta t}\left(e^{\Delta t A / 2} u_{0}\right)\right) d \theta \\
= & e^{\Delta t A} u_{0}+\Delta t e^{\Delta t A / 2} B\left(e^{\Delta t A / 2} u_{0}\right)+e_{2}
\end{aligned}
$$

where $e_{2}$ is given by (4.5).

In (4.3) we express the integrand by a formula of the type (4.2) by using

$$
G(u(\sigma))=G\left(e^{\sigma A} u_{0}\right)+\int_{0}^{\sigma} d G\left(e^{(\sigma-\tau) A} u(\tau)\right)\left[e^{(\sigma-\tau) A} B(u(\tau))\right] d \tau
$$

for the last part of the integrand in (4.3), with

$$
G(v)=G_{s, \sigma}(v)=d B\left(e^{(s-\sigma) A} v\right)\left[e^{(s-\sigma) A} B(v)\right]
$$

and 


$$
\begin{aligned}
d G(v)[w]= & d^{2} B\left(e^{(s-\sigma) A} v\right)\left[e^{(s-\sigma) A} w, e^{(s-\sigma) A} B(v)\right] \\
& +d B\left(e^{(s-\sigma) A} v\right)\left[e^{(s-\sigma) A} d B(v)[w]\right] .
\end{aligned}
$$

Then we obtain,

$$
\begin{aligned}
e_{1}= & \int_{0}^{\Delta t} \int_{0}^{s} e^{(\Delta t-s) A} d B\left(e^{s A} u_{0}\right)\left[e^{(s-\sigma) A} B\left(e^{s A} u_{0}\right)\right] d \sigma d s \\
& +\int_{0}^{\Delta t} \int_{0}^{s} \int_{0}^{\sigma} d G_{s, \sigma}\left(e^{(\sigma-\tau) A} u(\tau)\right)\left[e^{(\sigma-\tau) A} B(u(\tau))\right] d \tau d \sigma d s .
\end{aligned}
$$

We return to the error formula (4.6) and write the principal error term

$$
\Delta t e^{\Delta t A / 2} B\left(e^{\Delta t A / 2} u_{0}\right)-\int_{0}^{\Delta t} e^{(\Delta t-s) A} B\left(e^{s A} u_{0}\right) d s
$$

in second-order Peano form

$$
\Delta t f\left(\frac{1}{2} \Delta t\right)-\int_{0}^{\Delta t} f(s) d s=\Delta t^{3} \int_{0}^{1} \kappa_{2}(\theta) f^{\prime \prime}(\theta \Delta t) d \theta
$$

with the second-order Peano kernel $\kappa_{2}$ of the midpoint rule and $f$ of (4.7). We have

$$
f^{\prime \prime}(s)=e^{(\Delta t-s) A}[A,[A, B]]\left(e^{s A} u_{0}\right) .
$$

The double commutator reads

$$
\begin{aligned}
{[A,[B, A]](v)=} & {[A, d A B(v)-d B(v) A(v)](v) } \\
= & d A^{2} B(v)-d A d B(v) A(v)-d^{2} A B(v) A(v) \\
& \quad-d A d B(v) A(v)+d^{2} B(A(v))^{2}+d B(v) d A A(v) \\
= & A^{2}(B(v))-2 A(d B(v) A(v))+d^{2} B(A(v))^{2}+d B(v) A^{2}(v) \\
= & A^{2}\left(v v_{x}\right)-2 A(v A v)_{x}+\left((A v)^{2}\right)_{x}+\left(v A^{2} v\right)_{x},
\end{aligned}
$$

since $d^{2} A=0$. The operator $A$ is linear in $\partial_{x}^{\ell}$, and the highest order derivative in the term $\left((A v)^{2}\right)_{x}$ is $\ell+1 \leq 2 \ell-1$, so we consider

$$
\begin{aligned}
& \partial_{x}^{2 \ell}\left(v v_{x}\right)-2 \partial_{x}^{\ell+1}\left(v \partial_{x}^{\ell} v\right)+\left(v \partial_{x}^{2 \ell} v\right)_{x} \\
& \quad=\sum_{j=0}^{2 \ell}\left(\begin{array}{c}
2 \ell \\
j
\end{array}\right) \partial_{x}^{2 \ell-j} v \partial_{x}^{j+1} v-2 \sum_{j=0}^{\ell+1}\left(\begin{array}{c}
\ell+1 \\
j
\end{array}\right) \partial_{x}^{\ell+1-j} v \partial_{x}^{\ell+j} v+v_{x} \partial_{x}^{2 \ell} v+v \partial^{2 \ell+1} v .
\end{aligned}
$$

We note that in the above sum, the terms containing derivatives of order $2 \ell+1$ and $2 \ell$ disappear, so

$$
\left\|f^{\prime \prime}(s)\right\|_{H^{r}} \leq C\left\|u_{0}\right\|_{H^{p}}^{2} .
$$

Now for the difference of (4.3) and (4.5),

$$
e_{2}-e_{1}=\frac{1}{2} \Delta t^{2} g\left(\frac{1}{2} \Delta t, \frac{1}{2} \Delta t\right)-\int_{0}^{\Delta t} \int_{0}^{s} g(s, \sigma) d \sigma d s+\tilde{e}_{2}-\tilde{e}_{1},
$$

where

$$
g(s, \sigma)=e^{(\Delta t-s) A} d B\left(e^{s A} u_{0}\right) e^{(s-\sigma) A} B\left(e^{\sigma A} u_{0}\right)
$$

and

$$
\tilde{e}_{1}=\int_{0}^{\Delta t} \int_{0}^{s} \int_{0}^{\sigma} d G_{s, \sigma}\left(e^{(\sigma-\tau) A} u(\tau)\right) e^{(\sigma-\tau) A} B(u(\tau)) d \tau d \sigma d s
$$


and

$$
\tilde{e}_{2}=\Delta t^{3} \int_{0}^{1} \frac{1}{2}(1-\theta)^{2} e^{\Delta t A / 2}\left(d^{2} B(B, B)+d B d B B\right)\left(\Phi_{B}^{\theta \Delta t}\left(u_{0}\right)\right) d \theta .
$$

To estimate the remainder terms $\tilde{e}_{i}$ we calculate

$$
\begin{aligned}
\left\|d G_{s, \sigma}(v) w\right\|_{H^{r}} \leq & \left\|d^{2} B\left(e^{(s-\sigma) A} B(v), w\right)\right\|_{H^{r}} \\
& +\left\|d B\left(e^{(s-\sigma) A} v\right) e^{(s-\sigma) A} d B(v) w\right\|_{H^{r}} \\
\leq & C\left(\|B(v)\|_{H^{r+1}}\|w\|_{H^{r+1}}+\|v\|_{H^{r+1}}\|d B(v) w\|_{H^{r+1}}\right) \\
\leq & C\left(\|v\|_{H^{r+2}}^{2}\|w\|_{H^{r+1}}+\|v\|_{H^{r+1}}\|v\|_{H^{r+2}}\|w\|_{H^{r+2}}\right) \\
\leq & C\|v\|_{H^{r+2}}^{2}\|w\|_{H^{r+2}}
\end{aligned}
$$

and

$$
\begin{aligned}
\left\|\left(d^{2} B(B, B)+d B d B B\right)(v)\right\|_{H^{r}} & \leq\left\|d^{2} B(B(v), B(v))\right\|_{H^{r}}+\|d B(v) d B(v) B(v)\|_{H^{r}} \\
& \leq C\left(\|B(v)\|_{H^{r+1}}^{2}+\|v\|_{H^{r+1}}\|d B(v) B(v)\|_{H^{r+1}}\right) \\
& \leq C\left(\|v\|_{H^{r+2}}^{4}+\|v\|_{H^{r+2}}^{2}\|B(v)\|_{H^{r+2}}\right) \\
& \leq C\|v\|_{H^{r+3}}^{4} .
\end{aligned}
$$

Then, using Lemma 3.1, the remainder terms are bounded by

$$
\left\|\tilde{e}_{1}\right\|_{H^{r}}+\left\|\tilde{e}_{2}\right\|_{H^{r}} \leq C \Delta t^{3}\left(\left\|u_{0}\right\|_{H^{p}}^{4}+\left\|u_{0}\right\|_{H^{p}}^{3}\right)
$$

since $2 \ell-1 \geq 3$.

The first two terms in $e_{2}-e_{1}$ are the quadrature error of a first-order twodimensional quadrature formula, which is bounded by

$$
\begin{aligned}
& \left\|\frac{1}{2} \Delta t^{2} g\left(\frac{1}{2} \Delta t, \frac{1}{2} \Delta t\right)-\int_{0}^{\Delta t} \int_{0}^{s} g(s, \sigma) d \sigma d s\right\|_{H^{r}} \\
& \leq C \Delta t^{3}\left(\max \|\partial g / \partial s\|_{H^{r}}+\max \|\partial g / \partial \sigma\|_{H^{r}}\right)
\end{aligned}
$$

where the maxima are taken over the triangle $\{(s, \sigma): 0 \leq \sigma \leq s \leq \Delta t\}$. In order to estimate the partial derivatives we write

$$
g(s, \sigma)=e^{(\Delta t-s) A} d B(v(s)) w(s, \sigma),
$$

where

$$
v(s)=e^{s A} u_{0} \text { and } w(s, \sigma)=e^{(s-\sigma) A} B(v(\sigma)) .
$$

With this notation

$$
\begin{aligned}
\frac{\partial g}{\partial s} & =e^{(\Delta t-s) A}\left(-A d B(v(s)) w(s, \sigma)+d^{2} B(A v(s), w(s, \sigma))+d B(v(s)) A w(s, \sigma)\right) \\
& =e^{(\Delta t-s) A}(-A(v(s) w(s, \sigma))+A v(s) w(s, \sigma)+v(s) A w(s, \sigma))_{x} .
\end{aligned}
$$

Since the flow determined by $A$ does not increase the Sobolev norms, it suffices to estimate the $H^{r+1}$ norm of $-A(v w)+(A v) w+v A w$. We see that the derivatives of order $\ell$ vanish, so that

$$
\left\|\frac{\partial g}{\partial s}\right\|_{H^{r}} \leq C\|v(s)\|_{H^{r+\ell}}\|w(s, \sigma)\|_{H^{r+\ell}} \leq C\left\|u_{0}\right\|_{H^{r+\ell}}\left\|u_{0}\right\|_{H^{r+\ell+1}}^{2} \leq C\left\|u_{0}\right\|_{H^{p}}^{3},
$$


since $\ell \geq 2$. For the other partial derivative, we get

$$
\frac{\partial g}{\partial \sigma}=e^{(\Delta t-s) A} d B(v(s))\left(e^{(s-\sigma) A}(-A B(v(s))+d B(v(\sigma)) A v(\sigma))\right),
$$

so that

$$
\left\|\frac{\partial g}{\partial \sigma}\right\|_{H^{r}} \leq C\|v(s)\|_{H^{r+1}}\|-A B(v(s))+d B(v(\sigma)) A v(\sigma)\|_{H^{r+1}} .
$$

The last factor is $-A\left(v v_{x}\right)+(v A v)_{x}$, and again the derivatives of order $\ell+1$ vanish, so that

Therefore we obtain

$$
\left\|\frac{\partial g}{\partial \sigma}\right\|_{H^{r}} \leq C\left\|u_{0}\right\|_{H^{p}}^{3}
$$

$$
\left\|e_{2}-e_{1}\right\|_{H^{r}} \leq C \Delta t^{3}\left(\left\|u_{0}\right\|_{H^{p}}^{4}+\left\|u_{0}\right\|_{H^{p}}^{3}\right),
$$

which together with the bound for the quadrature error of the midpoint rule for $f$ yields the stated result.

\section{Proof of Theorem 2.2}

We use "Lady Windermere's fan" in $H^{r}$ in combination with the $H^{p}$ bound of $u_{n}$ from Theorem 2.1 and the local error bound from Lemma 6.1, For $t_{n}=n \Delta t \leq T$ we have, as in the proof of Theorem 2.1 .

$$
\begin{aligned}
\left\|u_{n}-u\left(t_{n}\right)\right\|_{H^{r}} & \leq \sum_{k=0}^{n-1}\left\|\Phi^{(n-k-1) \Delta t}\left(\Psi^{\Delta t}\left(u_{k}\right)\right)-\Phi^{(n-k-1) \Delta t}\left(\Phi^{\Delta t}\left(u_{k}\right)\right)\right\|_{H^{r}} \\
& \leq \sum_{k=0}^{n-1} K(R, T)\left\|\Psi^{\Delta t}\left(u_{k}\right)-\Phi^{\Delta t}\left(u_{k}\right)\right\|_{H^{r}} \\
& \leq \sum_{k=0}^{n-1} K(R, T) c_{2}\left(C_{0}\right) \Delta t^{3} \leq K(R, T) c_{2}\left(C_{0}\right) T \Delta t^{2} .
\end{aligned}
$$

This completes the proof of Theorem 2.2

\section{REFERENCES}

[1] A. Ambrosetti and G. Prodi. A Primer of Nonlinear Analysis. Cambridge UP, Cambridge, 1995.

[2] D. J. Benney. Long waves on liquid films. J. Math. and Phys. 45:150-155 (1966). MR.0201125 $(34: 1010)$

[3] H. A. Biagioni and F. Linares. On the Benney-Lin and Kawahara equations. J. Math. Anal. Appl. 211:131-152 (1997). MR.1460163 (98e:35140)

[4] J. L. Bona and R. Smith. The initial-value problem for the Korteweg-de Vries equation. Philos. Trans. Roy. Soc. London Ser. A 278:555-601 (1975). MR0385355 (52:6219)

[5] E. Hairer, S. P. Nørsett, and G. Wanner. Solving Ordinary Differential Equations I. Nonstiff Problems. Second edition. Springer, Berlin, 1993. MR.1227985 (94c:65005)

[6] H. Holden, K. H. Karlsen, K.-A. Lie, and N. H. Risebro. Splitting for Partial Differential Equations with Rough Solutions. European Math. Soc. Publishing House, Zürich, 2010. MR.2662342 (2011j:65002)

[7] H. Holden, K. H. Karlsen, and N. H. Risebro. Operator splitting methods for generalized Korteweg-de Vries equations. J. Comput. Phys. 153:203-222 (1999). MR.1703652 (2001c:65101)

[8] H. Holden, K. H. Karlsen, N. H. Risebro, and T. Tao. Operator splitting methods for the Korteweg-de Vries equation. Math. Comp. 80:821-846 (2011). MR2772097 
[9] H. Holden, K. H. Karlsen, T. Karper. Operator splitting for two-dimensional incompressible fluid equations. Math. Comp., to appear.

[10] T. Jahnke and C. Lubich. Error bounds for exponential operator splittings. BIT 40:735-744 (2000). MR 1799313 (2001k:65143)

[11] Y. Jia and Z. Huo. Well-posedness for the fifth-order shallow water equations. J. Diff. Eqn. 246:2448-2467 (2009). MR2498848(2010k:35411)

[12] T. Kawahara. Oscillatory solitary waves in dispersive media. J. Phys. Soc. Japan 33:260-264 (1975).

[13] C. E. Kenig, G. Ponce, and L. Vega. Well-posedness of the initial value problem for the Korteweg-de Vries equation. J. Amer. Math. Soc. 4:323-347 (1991). MR1086966 (92c:35106)

[14] O. A. Ladyženskaja, V. A. Solonnikov, and N. N. Ural'ceva. Linear and Quasi-linear Equations of Parabolic Type. American Mathematical Society, Providence, 1968. MR0241821 $(39: 3159 \mathrm{a})$

[15] S. P. Lin. Finite amplitude side-band stability of a viscous film. J. Fluid. Mech. 63:417-429 (1974).

[16] F. Linares and G. Ponce. Introduction to Nonlinear Dispersive Equations. Springer, 2009. MR2492151(2010j:35001)

[17] C. Lubich. On splitting methods for Schrödinger-Poisson and cubic nonlinear Schrödinger equations. Math. Comp. 77:2141-2153 (2008). MR2429878 (2009d:65114)

[18] T. Tao. Nonlinear Dispersive Equations. Local and Global Analysis. Amer. Math. Soc., Providence, 2006. MR2233925(2008i:35211)

[19] F. Tappert. Numerical solutions of the Korteweg-de Vries equation and its generalizations by the split-step Fourier method. In: (A. C. Newell, editor) Nonlinear Wave Motion, Amer. Math. Soc., 1974, pp. 215-216.

Department of Mathematical Sciences, Norwegian University of Science and Technology, NO-7491 Trondheim, Norway, - and - Centre of Mathematics for Applications, University of Oslo, P.O. Box 1053, Blindern, NO-0316 Oslo, Norway

E-mail address: holden@math.ntnu.no

$U R L:$ http://www.math.ntnu.no/ ${ }^{\text {holden }}$

Mathematisches Institut, Universität Tübingen, Auf der Morgenstelle 10, D-72076 Tübingen, Germany

E-mail address: lubich@na.uni-tubingen.de

$U R L:$ http://na.uni-tuebingen.de/ lubich/

Centre of Mathematics for Applichtions, University of Oslo, P.O. Box 1053, BlinDERN, NO-0316 OSLO, NoRWAY

E-mail address: nilshr@math.uio.no

$U R L$ : folk.uio.no/nilshr 